\title{
Disease activity, resilience and health- related quality of life in Chinese patients with rheumatoid arthritis: a multi-center, cross-sectional study
}

\author{
Li Liu', Xin Xu' ${ }^{2}$ Neili Xu ${ }^{3}$ and Lie Wang ${ }^{1 *}$
}

\begin{abstract}
Background: Positive psychological constructs that can moderate or mediate the negative impact of disease activity on health-related quality of life (HRQOL) in patients with rheumatoid arthritis (RA) have not been explored widely. This study aimed to assess the associations of disease activity, resilience with HRQOL and the moderating and mediating roles of resilience among Chinese RA patients.
\end{abstract}

Methods: A multi-center, cross-sectional study was conducted in RA inpatients in northeast of China. A total 298 subjects completed the Medical Outcomes Study 36-item Short-Form Health Survey (SF-36) and Ego-Resiliency Scale (ERS) to measure HRQOL and resilience. For the SF-36, physical function, physical role limitation, bodily pain and general health perception are gathered into physical component summary (PCS), while vitality, social functioning, emotional role limitation and mental health are gathered into mental component summary (MCS). Disease activity was evaluated by the Disease Activity Score 28-C-reactive protein (DAS28-CRP). Hierarchical regression analysis was applied to examine the associations of disease activity, resilience and the disease activity*resilience interaction with PCS and MCS, respectively. Asymptotic and resampling strategies were utilized to examine the mediating role of resilience.

Results: The mean scores of PCS and MCS were 40.67 and 59.14, respectively. Disease activity was negatively associated with both PCS and MCS, and resilience was only positively associated with MCS. The disease

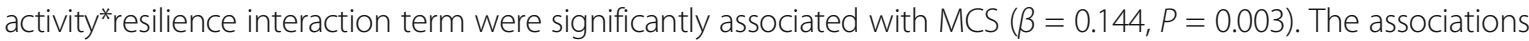
between disease activity and MCS were gradually reduced in low (1 SD below the mean, $\beta=-0.369, P<0.001$ ), mean $(\beta=-0.218, P<0.001$ ) and high (1 SD above the mean, $\beta=-0.068, P=0.369$ ) groups of resilience. Resilience acted as a partial mediator in the disease activity-MCS association (effect size was -0.085 , BCa 95\% Cl: $-0.159,-0.028$ ).

Conclusions: Disease activity was negatively associated with both physical and mental HRQOL, and resilience was only positively associated with mental HRQOL. Resilience could attenuate and mediate the association between disease activity and mental HRQOL. In addition to controlling disease activity, targeted intervention strategies designed for resilience should be strengthened to improve the HRQOL of this population.

Keywords: Health-related quality of life, SF-36, DAS28-CRP, Positive psychology, Rheumatoid arthritis

\footnotetext{
* Correspondence: liewang@cmu.edu.cn

${ }^{1}$ Department of Social Medicine, School of Public Health, China Medical

University, No.77 Puhe Road, Shenyang North New Area, Shenyang, Liaoning

110122, People's Republic of China

Full list of author information is available at the end of the article
} 


\section{Background}

Rheumatoid arthritis (RA) is a chronic, symmetric and progressive inflammatory disease that includes some disease-specific symptoms such as joint pain, stiffness, swelling and fatigue [1]. RA can lead to disability and significantly interfere with functional adaptation [1]. RAphysical and functional stressors together create enormous psychological distress. RA patients often have a high prevalence of emotional stress, depression and anxiety [2]. Thus, considerable physical and psychological challenges can seriously damage these patients' overall health-related quality of life (HRQOL) [3, 4]. In general, HRQOL refers to the ways in which a given health condition affects a patient's physical ability and capacity to function in various social and emotional roles, including physical functioning and mental well-being [5].

Certainly, it is important to identify factors that can influence HRQOL to make it a more feasible target for clinical management. According to the Wilson and Cleary model (WCM) [6], six categories are directly or indirectly related to patients' overall HRQOL: biological and physiological status, symptom status, functional status, general health perceptions, individual characteristics, and environmental characteristics. In the WCM, a causal pathway between biological and physiological status and HRQOL is proposed through sequential effects on symptoms, functions and general health perceptions [6]. Therefore, biological and physiological status can play prominent roles in explaining physical functioning and mental well-being in RA patients [7, 8].

Disease activity is one of the indicators of the clinical assessment of biological and physiological status to guide treatment adjustment in RA [9]. Previous qualitative studies have shown a strong relationship between disease activity and HRQOL including physical and mental components $[7,8,10,11]$. In addition, a common observation has been reported that there is a significant variability in health functioning among RA patients who have similar levels of disease activity [10,11]. The finding gives a strong hint that there are some individual and environmental factors that play a role in mediating or moderating the relationship between disease activity and HRQOL in RA patients [10]. Therefore, those potential mediators and moderators are necessary to be identified for improving HRQOL promptly through targeted intervention, while trying to reduce the disease activity of RA. However, the contribution of such factors to HRQOL has not been adequately determined in RA patients.

With the rise of positive psychology, researchers have gradually shifted attention to the exploration of the effects of positive psychological constructs on health outcomes in patients with chronic diseases [12, 13]. Positive psychological capacities seem not only to be associated with good physical and mental outcomes in those patients, but also to play a vital role in re-realizing their conditions [14]. In clinical practice, several interventions integrating positive psychological capacities have shown encouraging health outcomes $[15,16]$. Among these positive psychological constructs, resilience is a focus in this field. With regard to definition, resilience generally refers to the dynamic capacity of someone to bounce back from life adversities to maintain or recuperate his health successfully [17]. Rutter proposed four possible mechanisms of resilience: (1) reducing the impact of risk factors, including changes in individuals' cognition about risk factors, and avoiding or reducing contact with them; (2) reducing the negative chain reaction of negative life events; (3) improving the levels of self-esteem and selfefficacy; (4) helping individuals access available resources for hope and success [17]. In view of the results of previous studies, resilience can directly indicate lower levels of perceived distress, better adjustment and health outcomes among patients with chronic diseases, such as RA, cancer, diabetes and psychiatric disorders [18-21]. Moreover, resilience can also act as a 'moderator' to enhance or attenuate the effects of stressors on physical and mental well-being. Rainone et al. found that there was a moderating role of resilience on the relationship between affective disorders and HRQOL for adolescents and young adults with multiple sclerosis [22]. Min et al. reported that resilience played moderation on the negative effect of pain on depression and post-traumatic growth in individuals with spinal cord injury [23]. In addition, previous researches indicated that resilience played a mediating role in the relationship between cancer symptom distress and HRQOL $[24,25]$. Thus, resilience probably could help explain individual variation in HRQOL in patients with chronic diseases. However, the positive role of resilience on HRQOL, and whether or not resilience moderates or mediates the relationship between disease-related physiological status and HRQOL have not been examined among Chinese RA patients to our best knowledge.

In light of the above concerns, the present study aimed to assess the effects of disease activity and resilience on HRQOL, and to examine the moderating and mediating roles of resilience on the association between disease activity and HRQOL in Chinese RA patients.

\section{Methods}

\section{Ethics statement}

The study was approved by the Ethics Committee on Human Experimentation of Shengjing Hospital of China Medical University, Central Hospital of Benxi, General Hospital of Fushun Mining Bureau and Sujiatun Central Hospital of Shenyang, and was conducted in accordance with the principles contained in the Declaration of Helsinki for studies in humans. All the patients provided 
their written informed consent after being informed about the objectives and procedures of the study, and they participated in the study voluntarily and anonymously.

\section{Study design and sample}

This was a multi-center and cross-sectional study. From December 2014 through January 2016, successive inpatients diagnosed with RA were recruited at the Department of Rheumatology at Shengjing Hospital of China Medical University, Central Hospital of Benxi, General Hospital of Fushun Mining Bureau and Sujiatun Central Hospital of Shenyang. The four medical centers gather a substantial numbers of patients in Liaoning Province that is in the northeast of China.

In this study, the inclusion criteria of patient recruitment included: (1) was diagnosed with RA according to the ACR/EULAR 2010 classification criteria; (2) was at least 18 years old or older; (3) could understand and communicate in Chinese well. The exclusion criteria of patient recruitment were: (1) had a psychiatric history in the past; (2) had intellectual and/or cognitive impairments; (3) was suffering from other severe comorbidities simultaneously. Rheumatologists-in-charge requested all eligible patients to participate in the study. A set of selfadministered questionnaires was distributed to the participants after obtaining the patients' written consent. Clinical information was gathered from medical record.

In total, 325 patients were recruited as potential subjects. Twelve patients refused to cooperate subsequently. Thus, the questionnaires were distributed to 313 eligible patients, and 15 responses with missing data concerning any item in the questionnaires were excluded from the final analysis. In the final, effective responses were received from 298 patients (91.7\%).

\section{Measures}

\section{Measurement of HRQOL}

HRQOL was measured with the Chinese version of the Medical Outcomes Study 36-item Short-Form Health Survey (SF-36), consisting of 36 items that measured eight dimensions: physical function, role limitations related to physical problems, bodily pain, general health perception, vitality, social functioning, role limitations due to emotional problems, and mental health [5]. These dimensions were gathered into physical component summary (PCS) and mental component summary (MCS), respectively. The raw score of each component summary is converted to a standard score ranging from 0 to 100 , with a higher score indicating better HRQOL perception. The SF-36 has good reliability and validity among various Chinese patients $[19,26]$. In this study, the Cronbach's $\alpha$ values for the PCS and MCS subscales were 0.89 and 0.88 , respectively.

\section{Measurement of disease activity}

The disease activity of RA was indicated by the Disease Activity Score 28-C-reactive protein (DAS28-CRP) [27]. On the basis of swollen joint counts (SJC), tender joint counts (TJC) and the level of CRP, DAS28-CRP was calculated using the formula: DAS28-CRP $=[0.56$ *sqrt(TJC28) $+0.28 *$ sqrt(SJC28) $+0.36 * \ln (\mathrm{CRP}+1)]^{*} 1.10+1.15$. Also, disease activity was divided into four groups based on the cut-off points of DAS28-CRP: clinical remission < 2.6, low level 2.6-3.2, moderate level 3.2-5.1, and high level $>5.1$.

\section{Measurement of resilience}

Resilience was measured by the Chinese version of EgoResiliency Scale (ERS) [28], which consists of 14 items as a one-dimensional scale. The ERS emphasizes individual's psychological capacity to bounce back especially experiencing overwhelming or stressful situations. Each item is scored on a 4-point Likert scale in accordance with the patients' personal feelings about resilience, ranging from 1 'does not apply at all' to 4 'completely apply'. The summed score ranges from 14 to 56 , which indicates the higher the score is, the more swiftly recovery individuals make from those negative situations. The ERS fits for a variety of patients with high validity and reliability in China $[29,30]$, and the Cronbach's $\alpha$ value for the ERS was 0.88 in this study.

\section{Demographic characteristics}

Demographic characteristics included gender, age, marital status, educational level, employment, household monthly income and residence. Marital status was categorized as married/cohabited and single/divorced/ widowed/separated. Educational level was classified as junior high school or below, senior high school and junior college or above. Employment was sorted into two groups as unemployment and part time/full time. Household monthly income (RMB) was categorized as $<3000,<4000$ and $\geq 4000$ yuan. Residence was divided into urban and rural.

\section{Clinical variables}

Clinical variables including TJC, SJC, CRP, duration of suffering from RA, anemia, early morning stiffness (EMS), and chronic comorbidities were collected. The duration of suffering RA was classified as: $\leq 1,2-5,6-$ 10 and $>10$ years. Anemia was categorized as yes or no. EMS was divided into three groups: no, $\leq 1$ and $>1 \mathrm{~h}$. If the respondents were suffering from other severe comorbidities, such as osteoarthritis, sclerosis, hypertension, coronary heart disease and diabetes, chronic comorbidity was defined as yes; otherwise, it was defined as no. 


\section{Statistical analysis}

All variables were described with number (n), percentage (\%), mean, standard deviation (SD) and range appropriately. Group differences of continuous variables were examined using independent sample t-test or one way analysis of variance. Correlations among continuous variables were tested using Pearson's correlation analysis. Hierarchical regression analysis was adopted to examine the associations of disease activity and resilience with HRQOL, as well as to explore the moderating role of resilience on the association of disease activity with HRQOL. Besides gender and age, demographic and clinical variables related to HRQOL in univariate analysis $(P<0.15)$ were adjusted. In step 1 , gender, age, and potential control variables were added. Disease activity was added in step 2, and resilience was added in step 3. Ultimately, a disease activity*resilience interaction term was added in step 4. If the interaction effect was statistically significant, simple slope analyses were conducted to visualize the interaction term [31]. The variables in the models were centered before regression analysis. Asymptotic and resampling strategies were used to examine the mediating role of resilience in the association between disease activity and HRQOL. Biascorrected and accelerated 95\% confidence intervals (BCa 95\% CI) for mediation were conducted with the bootstrap estimate on a basis of 5000 bootstrap samples, in which exclusion of 0 indicated a significant mediating role [31]. Statistical analysis was executed by SPSS 19.0 software and a two-tailed $P<0.05$ was viewed as statistically significant.

\section{Results}

\section{Descriptive statistics}

Demographic and clinical characteristics and group differences in the PCS and MCS of HRQOL are displayed in Table 1. Of these subjects, $77.2 \%$ (230) were women, $88.9 \%$ (265) were married/cohabited, and they reported higher MCS scores $(t=2.324, P=0.021)$ than those single/divorced/widowed/separated subjects. There were $67.1 \%$ (200) subjects received a senior high school or above education, 61.1\% (182) were unemployment, 228 (76.5\%) had a household monthly income level of $<4000$ yuan RMB, and 217 (72.8\%) lived in urban areas. With regard to clinical variables, 30 (10.1\%) subjects' DAS28CRP scores were below 2.6, 89 (29.9\%) had a RA duration of $\leq 1$ year, 120 (40.3\%) had anemia, 214 (71.8\%) suffered from EMS, and 190 (63.8\%) had at least one other chronic disease. DAS28-CRP and EMS were significantly related to both PCS and MCS, and patients with anemia reported lower PCS than those without anemia $(t=3.422, P=0.001)$. However, statistically significant relations between PCS and MCS and the other demographic and clinical variables were not found in this study.

The levels of PCS, MCS, age, DAS28-CRP, resilience are provided in Table 2. The mean scores of PCS and MCS were $40.67(S D=10.86)$ and 59.14 $(S D=10.23)$, which ranged from 7.78 to 75.35 and from 29.06 to 83.75 , respectively. The mean values were 57.27 $(\mathrm{SD}=12.11), 4.57(\mathrm{SD}=1.52)$ and $34.22(\mathrm{SD}=5.50)$ for age, DAS28-CRP and resilience, respectively.

\section{Correlations among continuous variables}

The correlations among age, DAS28-CRP, resilience, PCS and MCS are displayed in Table 3. Both age and DAS28CRP were negatively correlated with PCS and MCS, respectively. Resilience was positively correlated with MCS $(r=0.530, P<0.01)$, and DAS28-CRP was negatively correlated with resilience $(r=-0.136, P<0.05)$.

\section{Hierarchical regression analysis}

The results of hierarchical regression analysis on PCS are displayed in Table 4. In step 1, the linear combination of demographic and medical control variables (gender, age, marital status, residence, anemia and EMS) significantly predicted PCS $(F=4.128$, adjusted $\left.R^{2}=0.069, P<0.01\right)$. DAS28-CRP was significantly and negatively associated with PCS in step $2(\beta=-0.374$, $P<0.001)$, and improved model fit $(F=8.781$, adjusted $\left.R^{2}=0.173, \Delta \mathrm{R}^{2}=0.105, P<0.01\right)$. In step 3 , DAS28CRP was also significantly and negatively associated with PCS $(\beta=-0.377, P<0.001)$, whereas resilience was not significantly associated with PCS $(\beta=-0.016, P=0.773)$. In step 4 , the disease activity*resilience interaction term was not significantly associated with PCS $(\beta=0.078$, $P=0.155)$. Then, the statistical power of the analysis was determined using post-hoc statistical power analysis on the basis of the sample size and the observed $R^{2}$, assuming $\alpha=0.05$. It indicated a statistical power of 99.99\% for the present sample.

The results of hierarchical regression analysis on MCS are displayed in Table 5. In step 1, the linear combination of demographic and medical control variables also significantly predicted MCS $(F=3.849$, adjusted $\left.R^{2}=0.063, P<0.01\right)$. DAS28-CRP was significantly and negatively associated with MCS in step $2(\beta=-0.308$, $P<0.001)$, and improved model fit $(F=6.674$, adjusted $\left.R^{2}=0.133, \Delta R^{2}=0.071, P<0.01\right)$. In step 3 , DAS28CRP was also significantly and negatively associated with $\operatorname{MCS}(\beta=-0.223, P<0.001)$, whereas resilience was significantly and positively associated with MCS $(\beta=0.469$, $P<0.001)$ and explained an additional $20.6 \%$ of the variance $\left(F=18.115\right.$, adjusted $R^{2}=0.342, \Delta R^{2}=0.206$, $P<0.01$ ). In step 4 , the disease activity*resilience interaction term was significantly and positively associated with $\operatorname{MCS}(\beta=0.144, P=0.003)$, and explained an 
Table 1 Demographic and clinical variables of participants in relation to the PCS and MCS of HRQOL

\begin{tabular}{|c|c|c|c|c|c|c|c|c|c|c|}
\hline \multirow[t]{2}{*}{ Variables } & \multirow[t]{2}{*}{$n$} & \multirow[t]{2}{*}{$\%$} & \multicolumn{2}{|l|}{ PCS } & \multirow[t]{2}{*}{$F / t$} & \multirow[t]{2}{*}{$P$} & \multicolumn{2}{|l|}{ MCS } & \multirow[t]{2}{*}{$F / t$} & \multirow[t]{2}{*}{$P$} \\
\hline & & & Mean & SD & & & Mean & SD & & \\
\hline Gender & & & & & 0.626 & 0.532 & & & 0.621 & 0.549 \\
\hline Men & 68 & 22.8 & 41.40 & 11.49 & & & 59.82 & 10.18 & & \\
\hline Women & 230 & 77.2 & 40.46 & 10.68 & & & 58.94 & 10.26 & & \\
\hline Marital status & & & & & 0.338 & 0.736 & & & 2.324 & 0.021 \\
\hline Single/divorced/widowed/separated & 33 & 11.1 & 41.28 & 11.27 & & & 55.27 & 11.15 & & \\
\hline Married/cohabited & 265 & 88.9 & 40.60 & 10.82 & & & 59.62 & 10.03 & & \\
\hline Educational level & & & & & 0.903 & 0.407 & & & 0.054 & 0.948 \\
\hline Junior high school or below & 98 & 32.9 & 39.74 & 10.20 & & & 59.21 & 9.34 & & \\
\hline Senior high school & 135 & 45.3 & 40.68 & 10.86 & & & 59.27 & 10.34 & & \\
\hline Junior college or above & 65 & 21.8 & 42.07 & 11.78 & & & 58.78 & 11.38 & & \\
\hline Employment & & & & & 0.418 & 0.676 & & & 1.312 & 0.191 \\
\hline Unemployment & 182 & 61.1 & 40.46 & 10.27 & & & 58.52 & 10.21 & & \\
\hline Part-time/full-time & 116 & 38.9 & 41.00 & 11.75 & & & 60.11 & 10.24 & & \\
\hline Household monthly income (yuan) & & & & & 1.880 & 0.154 & & & 1.045 & 0.353 \\
\hline$<3000$ & 107 & 35.9 & 39.14 & 10.68 & & & 60.25 & 9.99 & & \\
\hline$<4000$ & 121 & 40.6 & 41.91 & 9.74 & & & 58.73 & 10.26 & & \\
\hline$\geq 4000$ & 70 & 23.5 & 40.89 & 12.67 & & & 58.17 & 10.53 & & \\
\hline Residence & & & & & 1.806 & 0.072 & & & 0.016 & 0.987 \\
\hline Urban & 217 & 72.8 & 41.36 & 10.52 & & & 59.15 & 10.42 & & \\
\hline Rural & 81 & 27.2 & 38.82 & 11.58 & & & 59.13 & 9.77 & & \\
\hline DAS28-CRP & & & & & 11.778 & $<0.001$ & & & 6.067 & 0.001 \\
\hline$<2.6$ & 30 & 10.1 & 47.79 & 10.82 & & & 63.96 & 9.97 & & \\
\hline $2.6-3.2$ & 26 & 8.7 & 42.99 & 9.88 & & & 61.25 & 9.92 & & \\
\hline $3.2-5.1$ & 142 & 47.7 & 41.82 & 10.52 & & & 59.88 & 10.13 & & \\
\hline$>5.1$ & 100 & 33.6 & 36.31 & 9.96 & & & 56.10 & 9.78 & & \\
\hline Duration (years) & & & & & 0.465 & 0.707 & & & 0.644 & 0.587 \\
\hline$\leq 1$ & 89 & 29.9 & 41.76 & 12.19 & & & 59.45 & 11.77 & & \\
\hline $2-5$ & 82 & 27.5 & 39.97 & 10.26 & & & 58.37 & 9.74 & & \\
\hline $6-10$ & 52 & 17.4 & 40.08 & 10.53 & & & 58.19 & 9.43 & & \\
\hline$>10$ & 75 & 25.2 & 40.56 & 10.12 & & & 60.29 & 9.35 & & \\
\hline Anemia & & & & & 3.422 & 0.001 & & & 1.493 & 0.137 \\
\hline Yes & 120 & 40.3 & 38.10 & 10.03 & & & 58.07 & 9.44 & & \\
\hline No & 178 & 59.7 & 42.41 & 11.07 & & & 59.87 & 10.70 & & \\
\hline EMS (hours) & & & & & 3.421 & 0.034 & & & 5.841 & 0.003 \\
\hline No & 84 & 28.2 & 42.66 & 10.93 & & & 59.15 & 10.56 & & \\
\hline$\leq 1$ & 164 & 55.0 & 40.58 & 10.58 & & & 60.44 & 9.66 & & \\
\hline$>1$ & 50 & 16.8 & 37.64 & 11.09 & & & 54.88 & 10.53 & & \\
\hline Chronic comorbidity & & & & & 0.236 & 0.814 & & & 0.964 & 0.336 \\
\hline No & 108 & 36.2 & 40.87 & 11.61 & & & 58.38 & 10.81 & & \\
\hline Yes & 190 & 63.8 & 40.56 & 10.43 & & & 59.57 & 9.89 & & \\
\hline
\end{tabular}

Abbreviations: $P C S$, physical component summary; MCS, mental component summary; HRQOL, health-related quality of life; SD, standard deviation; DAS28-CRP, Disease Activity Score 28-C-reactive protein; EMS, early morning stiffness 
Table 2 Descriptive statistics for continuous variables

\begin{tabular}{llll}
\hline Variables & Mean & SD & Range \\
\hline PCS & 40.67 & 10.86 & $7.78-75.35$ \\
MCS & 59.14 & 10.23 & $29.06-83.75$ \\
Age (years) & 57.27 & 12.11 & $21-82$ \\
DAS28-CRP & 4.57 & 1.52 & $1.56-8.13$ \\
Resilience & 34.22 & 5.50 & $23-56$ \\
\hline
\end{tabular}

Abbreviations: $S D$, standard deviation; $P C S$, physical component summary; $M C S$, mental component summary; DAS28-CRP, Disease Activity Score 28-C-reactive protein

additional $1.9 \%$ of the variance $(F=17.647$, adjusted $\left.R^{2}=0.359, \Delta R^{2}=0.019, P<0.01\right)$. Simple slope analysis revealed that when resilience was higher, the association between disease activity and MCS became weaker. In other words, the association between disease activity and MCS was gradually reduced in the low (1 SD below the mean, $\beta=-0.369, P<0.001)$, mean $(\beta=-0.218$, $P<0.001)$ and high (1 SD above the mean, $\beta=-0.068$, $P=0.369)$ groups of resilience. The interaction is visualized in Fig. 1. Furthermore, a reduced $\beta$ coefficient of disease activity in step 3 suggested that resilience could mediate the disease activity-MCS association.

\section{Resilience as a mediator in the association between disease activity and MCS}

With regard to the results of asymptotic and resampling strategies, disease activity was significantly and negatively associated with resilience $(\beta=-0.181, P=0.006)$. Because resilience was significantly and positively associated with MCS, a significant mediating role of resilience (effect size was -0.085 , BCa 95\% CI: $-0.159,-0.028$ ) in the association between disease activity and MCS was revealed. In addition, the proportion of mediating role of resilience was $27.6 \%$ in the total effect of disease activity on MCS.

\section{Discussion}

In this study, both the PCS and MCS of HRQOL in patients with RA were significantly decreased compared to the general population of China [32]. This finding is consistent with previous studies conducted across countries, showing that all aspects of HRQOL of RA patients are impaired [3, 8, 33-35]. Also in agreement with most prior researches $[7,8,10,34-37]$, the score of the PCS was lower than the MCS, suggesting that RA has a greater impact on physical health than mental wellbeing. When compared with RA patients from other studies in Asian population, patients in our sample from mainland China tended to show a lower level of PCS [8, 12, 26, 36, 37]. The level of MCS was consistent with the results of the three studies mentioned above $[8,26$, 37], but it was higher than the results from most previous studies across countries [7, 11, 12, 34-36, 38]. One important reason for the discrepancy in the MCS comparisons may be that considerable developments in treatment in recent years have had a significant beneficial effect on RA patients' HRQOL [39]. However, for the discrepancy in the PCS comparisons, the disease can become very severe and difficult to control by the time rheumatological care is accessed due to limited knowledge or self-management about RA in mainland China [40]. Other reasons may be some differences in the sources and cultural backgrounds of subjects, and the interpretation of the concept of HRQOL across studies. Furthermore, patients with RA have significantly reduced levels of both PCS and MCS in comparison to those with other diseases, such as ankylosing spondylitis, psoriatic arthritis, and systemic lupus erythematosus [26, $35,41]$. In view of the above facts, policymakers and healthcare workers need to raise awareness of the improvement of HRQOL in patients with RA, and more resources should be made available in China.

In addition, we found that disease activity had significant impacts on physical and mental HRQOL. Our results were consistent with the most existing literatures strongly associating disease activity with the HRQOL of RA patients $[7,8]$. This study extended the information available in the existing literature by showing that Chinese RA patients who experienced higher disease activity reported lower levels of HRQOL. RA patients in mainland China are prone to suffer from a higher disease activity due to the lack of early diagnosis, aggressive treatment and selfmanagement [40]. In consequence, they were more likely to experience uncertainty concerning treatment, which could profoundly affect their HRQOL. The significant direct effect of disease activity on the HRQOL of RA patients emphasizes the importance of regular disease treatment and management. Based on the clinical evaluation of disease activity, a timely adjustment of drug treatment could reduce the degree of disease activity, and improve HRQOL in RA patients.

Resilience has been expected to predict and evaluate HRQOL among patients with chronic diseases [19-21, $24,25]$. Resilience was seldom studied in RA [18], a consensus was reached that higher level of resilience was beneficial for patients with RA and targeted psychological intervention required more emphasis. Consistent with previous studies [20, 21], resilience was strongly and positively associated with mental HRQOL in this study. RA patients with high resilience scores enjoyed high mental HRQOL, because they could recover easily and quickly from setbacks in their disease conditions. In general, resilience can allow RA patients to address their physical health correctly and to maintain a relatively good whole health state, resulting in a better HRQOL.

To our knowledge, the study was the first to explore the moderating role of resilience on the association 
Table 3 Correlations among continuous variables

\begin{tabular}{lllll}
\hline Variables & 1 & 2 & 3 & 4 \\
\hline 1. Age & 1 & & & \\
2. DAS28-CRP & 0.103 & 1 & & \\
3. Resilience & -0.101 & $-0.136^{*}$ & 1 & \\
4. PCS & $-0.129^{*}$ & $-0.398^{* *}$ & 0.054 & 1 \\
5. MCS & $-0.140^{*}$ & $-0.294^{* *}$ & $0.530^{* *}$ & $0.587^{* *}$
\end{tabular}

${ }^{*}, P<0.05 ; * *, P<0.01$. Abbreviations: DAS28-CRP, Disease Activity Score 28-Creactive protein; $P C S$, physical component summary; $M C S$, mental component summary

between disease activity and HRQOL in RA patients. We found that disease activity and resilience interact with each other in their associations with mental HRQOL, but not physical HRQOL. In other words, resilience could attenuate the impact of disease activity on mental HRQOL. Moreover, psychosocial resilience should be hypothesized to moderate the association between extrinsic stressor and mental health according to Rutter's protective mechanisms [17]. Also in other mental health domains, the interactions of resilience with extrinsic stressors have been confirmed. For instance, Ding et al. and Wingo et al. found that resilience moderated the association of childhood trauma with depressive symptoms [42, 43]. Resilience was found to be a protective factor for depressive symptoms and also mitigated the effects of peer victimization on depressive symptoms among rural-to-urban migrant children in China [44].

Some review literatures have indicated that resilience is a common response to physical disease diagnosis or treatment $[45,46]$. The patients have an initial response that fits somewhere on the distress-resilience continuum; however, treatment or intervention experiences can modify the initial response through a process of recalibration. Johnston et al. has confirmed that resilience involves maintaining healthy levels of functioning following adversity and that it is a dynamic process not a personality trait. Therefore, in most previous studies, physical disease was either considered as an adversity leading to resilience or as a variable modifying the relationship between adversity and resilience. As a dynamic process, resilience varies across different disease stages [46]. Consistent with the above findings, we found that disease activity had a significantly negative impact on the level of resilience, indicating that poor physical condition could reduce the patient's positive psychological capacities. Thus, the associations among disease activity, resilience, and mental HRQOL were in agreement with our hypothesis, disease activity also had an indirect effect on mental HRQOL through reducing resilience. This finding is in accordance with previous studies that showed the mediating roles of resilience in the relationships between cancer symptom distress and HRQOL, perceived symptoms and HRQOL in persons with longterm physical disabilities, and family function and HRQOL among the elderly [24, 25, 47, 48].

In view of these findings, as a target, resilience should be improved by effective positive psychological interventions in RA patients for coping with the negative effect of disease activity on HRQOL, especially mental wellbeing. Previous studies have developed some different strategies for resilience, such as cognitive behavioral and

Table 4 Hierarchical regression for exploring the correlates of PCS

\begin{tabular}{|c|c|c|c|c|c|c|c|c|}
\hline \multirow[t]{2}{*}{ Variables } & \multicolumn{2}{|l|}{ Step 1} & \multicolumn{2}{|l|}{ Step 2} & \multicolumn{2}{|l|}{ Step 3} & \multicolumn{2}{|l|}{ Step 4} \\
\hline & $\bar{\beta}$ & $P$ & $\bar{\beta}$ & $P$ & $\bar{\beta}$ & $P$ & $\bar{\beta}$ & $P$ \\
\hline Gender & -0.081 & 0.156 & -0.092 & 0.089 & -0.093 & 0.086 & -0.101 & 0.062 \\
\hline Age & -0.166 & 0.005 & -0.118 & 0.036 & -0.119 & 0.035 & -0.129 & 0.023 \\
\hline Marital status & -0.011 & 0.843 & -0.004 & 0.938 & -0.002 & 0.971 & -0.001 & 0.991 \\
\hline Residence & -0.121 & 0.039 & -0.095 & 0.085 & -0.094 & 0.088 & -0.094 & 0.089 \\
\hline Anemia & 0.177 & 0.002 & 0.113 & 0.040 & 0.113 & 0.040 & 0.117 & 0.034 \\
\hline \multicolumn{9}{|l|}{ EMS } \\
\hline Dummy_1 & -0.079 & 0.228 & 0.059 & 0.370 & 0.061 & 0.356 & 0.053 & 0.428 \\
\hline Dummy_2 & -0.145 & 0.027 & 0.028 & 0.677 & 0.029 & 0.668 & 0.028 & 0.677 \\
\hline DAS28-CRP & & & -0.374 & $<0.001$ & -0.377 & $<0.001$ & -0.375 & $<0.001$ \\
\hline Resilience & & & & & -0.016 & 0.773 & -0.004 & 0.949 \\
\hline DAS28-CRP*Resilience & & & & & & & 0.078 & 0.155 \\
\hline F & 4.128 & & 8.781 & & 7.790 & & 7.239 & \\
\hline Adjusted $R^{2}$ & 0.069 & & 0.173 & & 0.171 & & 0.174 & \\
\hline$\Delta R^{2}$ & & & 0.105 & & $<0.001$ & & 0.006 & \\
\hline
\end{tabular}

Gender, women versus men; Marital status, married/cohabited versus single/divorced/widowed/separated; Residence, rural versus urban; Anemia, no versus yes; Dummy_1, EMS $\leq 1$ versus no; Dummy_2, EMS > 1 versus no. ${ }^{*}$ Indicated multiplication. Abbreviations: PCS, physical component summary; EMS, early morning stiffness; DAS28-CRP, Disease Activity Score $28-C$-reactive protein 
Table 5 Hierarchical regression for exploring the correlates of MCS

\begin{tabular}{|c|c|c|c|c|c|c|c|c|}
\hline \multirow[t]{2}{*}{ Variables } & \multicolumn{2}{|l|}{ Step 1} & \multicolumn{2}{|l|}{ Step 2} & \multicolumn{2}{|l|}{ Step 3} & \multicolumn{2}{|l|}{ Step 4} \\
\hline & $\beta$ & $P$ & $\beta$ & $P$ & $\beta$ & $P$ & $\beta$ & $P$ \\
\hline Gender & -0.065 & 0.257 & -0.074 & 0.182 & -0.036 & 0.451 & -0.052 & 0.275 \\
\hline Age & -0.125 & 0.035 & -0.086 & 0.137 & -0.057 & 0.254 & -0.076 & 0.130 \\
\hline Marital status & 0.139 & 0.015 & 0.145 & 0.008 & 0.080 & 0.099 & 0.082 & 0.084 \\
\hline Residence & -0.024 & 0.686 & -0.003 & 0.964 & -0.019 & 0.699 & -0.018 & 0.714 \\
\hline Anemia & 0.095 & 0.097 & 0.042 & 0.449 & 0.039 & 0.430 & 0.045 & 0.353 \\
\hline \multicolumn{9}{|l|}{ EMS } \\
\hline Dummy_1 & 0.062 & 0.342 & 0.176 & 0.009 & 0.110 & 0.062 & 0.094 & 0.107 \\
\hline Dummy_2 & -0.158 & 0.016 & -0.016 & 0.818 & -0.044 & 0.464 & -0.046 & 0.440 \\
\hline DAS28-CRP & & & -0.308 & $<0.001$ & -0.223 & $<0.001$ & -0.218 & $<0.001$ \\
\hline Resilience & & & & & 0.469 & $<0.001$ & 0.492 & $<0.001$ \\
\hline DAS28-CRP*Resilience & & & & & & & 0.144 & 0.003 \\
\hline$F$ & 3.849 & & 6.674 & & 18.115 & & 17.647 & \\
\hline Adjusted $R^{2}$ & 0.063 & & 0.133 & & 0.342 & & 0.359 & \\
\hline$\Delta R^{2}$ & & & 0.071 & & 0.206 & & 0.019 & \\
\hline
\end{tabular}

Gender, women versus men; Marital status, married/cohabited versus single/divorced/widowed/separated; Residence, rural versus urban; Anemia, no versus yes; Dummy_1, EMS $\leq 1$ versus no; Dummy_2, EMS > 1 versus no. ${ }^{*}$ Indicated multiplication. Abbreviations: MCS, mental component summary; EMS, early morning stiffness; DAS28-CRP, Disease Activity Score 28-C-reactive protein

mindfulness-based interventions [49] and communitybased meditative Tai Chi programme [50], and these interventions were effective in the context of HRQOL.

Several theoretical and practical strengths of this study should be highlighted. Theoretically, the study provided a preliminary evidence for the association between resilience and HRQOL, and the moderating and mediating roles of on the association between disease activity and HRQOL in Chinese RA patients. In practice, the low level of HRQOL in RA patients should be paid sufficient attention in China; in order to improve RA patients'
HRQOL, an integrated strategy should be implemented by using existing treatment and positive psychological intervention to reduce disease activity and develop resilience.

There are some limitations that should be illustrated in the present study. Firstly, due to the cross-sectional design of the study, it was unable to assess the causal relationships among study variables. It only provided a snapshot of the associations among disease activity, resilience and HRQOL in Chinese RA patients. Therefore, longitudinal study should be carried out further to verify



Fig. 1 Simple slope plot of interaction between disease activity and resilience on MCS. Low, 1 SD below the mean; High, 1 SD above the mean. The values of disease activity and resilience were centered before regression analysis. The range of the centered value of disease activity is displayed. The mean and SD of the centered value of disease activity were 0 and 1.52, respectively. Gender, age, marital status, residence, anemia and EMS were adjusted. Abbreviations: MCS, mental component summary; SD, standard deviation; EMS, early morning stiffness 
our findings. Secondly, resilience and HRQOL were measured using self-administered questionnaires. The association between resilience and HRQOL might be influenced due to possible recall and reporting bias. Some effective process control measures have been carried out to minimize possible common-method bias. Thirdly, the present study was only conducted in a province of northeast China. Thus, the generalization of our findings requires to be further studied in other population with different cultural backgrounds. Finally, no differences in demographic characteristics and clinical variables were tested between participants and those who refused participation. The multi-center design and adequate sample size in this research could provide a good representation of patients with RA.

\section{Conclusions}

Chinese RA patients experience impaired physical and mental HRQOL. Disease activity was negatively associated with both physical and mental HRQOL, and resilience was only positively associated with mental HRQOL. Resilience could mediate and attenuate the association between disease activity and mental HRQOL. In addition to controlling disease activity, targeted intervention strategies designed for resilience should be strengthened to improve the HRQOL of this population.

\section{Abbreviations \\ BCa 95\% Cl: Bias-corrected and accelerated 95\% confidence intervals; DAS28- CRP: Disease Activity Score 28-C-reactive protein; EMS: Early morning stiffness; ERS: Ego-Resiliency Scale; HRQOL: Health-related quality of life; MCS: Mental component summary; PCS: Physical component summary; RA: Rheumatoid arthritis; SD: Standard deviation; SF-36: Medical Outcomes Study 36-item Short-Form Health Survey; SJC: Swollen joint counts; TJC: Tender joint counts; WCM: Wilson and Cleary model}

\section{Acknowledgments}

The authors would like to thank all the inpatients who voluntarily participated in this study.

\section{Funding}

There were no funding sources.

\section{Availability of data and materials}

The datasets analyzed during the current study are available from the corresponding author on reasonable request.

\section{Authors' contributions \\ $L W$ and $L L$ designed the research. $L W$ and NX organized the investigation. LW was the corresponding author of the paper. $L L$ and XX carried out data analysis. LL wrote the paper. XX and NX provided guidance in results interpreting and paper writing. All authors read and approved the final manuscript.}

\section{Ethics approval and consent to participate}

All patients provided their informed written consent. The study was approved by the Ethics Committee on Human Experimentation of Shengjing Hospital of China Medical University, Central Hospital of Benxi, General Hospital of Fushun Mining Bureau and Sujiatun Central Hospital of Shenyang, and was conducted in accordance with the principles contained in the Declaration of Helsinki for studies in humans.
Consent for publication

Not applicable.

\section{Competing interests}

The authors declare that they have no competing interests.

\section{Publisher's Note}

Springer Nature remains neutral with regard to jurisdictional claims in published maps and institutional affiliations.

\section{Author details}

${ }^{1}$ Department of Social Medicine, School of Public Health, China Medical University, No.77 Puhe Road, Shenyang North New Area, Shenyang, Liaoning 110122, People's Republic of China. ${ }^{2}$ Department of Clinical Epidemiology, Shengjing Hospital of China Medical University, No.36 Sanhao Street, Heping District, Shenyang, Liaoning 110004, People's Republic of China. ${ }^{3}$ Department of Rheumatology, Shengjing Hospital of China Medical University, No.36 Sanhao Street, Heping District, Shenyang, Liaoning 110004, People's Republic of China.

Received: 25 April 2017 Accepted: 19 July 2017

Published online: 24 July 2017

\section{References}

1. Smolen JS, Aletaha D, McInnes IB. Rheumatoid arthritis. Lancet. 2016; 388(10055):2023-38.

2. Vriezekolk JE, van Lankveld WG, Geenen R, van den Ende CH. Longitudinal association between coping and psychological distress in rheumatoid arthritis: a systematic review. Ann Rheum Dis. 2011;70(7):1243-50.

3. Senra H, Rogers H, Leibach G, Altamar ML, Plaza SL, Perrin P, et al. Healthrelated quality of life and depression in a sample of Latin American adults with rheumatoid arthritis. Int J Rheum Dis. 2014; doi:10.1111/1756-185X.12412.

4. Wan SW, He HG, Mak A, Lahiri M, Luo N, Cheung PP, et al. Health-related quality of life and its predictors among patients with rheumatoid arthritis. Appl Nurs Res. 2016;30:176-83.

5. Ware JE Jr, Sherbourne CD. The MOS 36-item short-form health survey (SF-36). I. Conceptual framework and item selection. Med Care. 1992;30(6):473-83.

6. Wilson IB, Cleary PD. Linking clinical variables with health-related quality of life. A conceptual model of patient outcomes. JAMA. 1995;273(1):59-65.

7. Kojima M, Kojima T, Ishiguro N, Oguchi T, Oba M, Tsuchiya H, et al. Psychosocial factors, disease status, and quality of life in patients with rheumatoid arthritis. J Psychosom Res. 2009;67(5):425-31.

8. Gong G, Mao J. Health-related quality of life among Chinese patients with rheumatoid arthritis: the predictive roles of fatigue, functional disability, selfefficacy, and social support. Nurs Res. 2016;65(1):55-67.

9. Anderson J, Caplan L, Yazdany J, Robbins ML, Neogi T, Michaud K, et al. Rheumatoid arthritis disease activity measures: American College of Rheumatology recommendations for use in clinical practice. Arthritis Care Res (Hoboken). 2012;64(5):640-7.

10. Nicassio PM, Kay MA, Custodio MK, Irwin MR, Olmstead R, Weisman MH. An evaluation of a biopsychosocial framework for health-related quality of life and disability in rheumatoid arthritis. J Psychosom Res. 2011;71(2):79-85.

11. Uhm DC, Nam ES, Lee HY, Lee EB, Yoon Yl, Chai GJ. Health-related quality of life in Korean patients with rheumatoid arthritis: association with pain disease activity, disability in activities of daily living and depression. J Korean Acad Nurs. 2012;42(3):434-42.

12. Bao Y, Li L, Guan Y, Wang W, Liu Y, Wang P, et al. Prevalence and associated positive psychological variables of anxiety and depression among patients with central nervous system tumors in China: a cross-sectional study. Psychooncology. 2017;26(2):262-9.

13. DuBois CM, Lopez OV, Beale EE, Healy BC, Boehm JK, Huffman JC. Relationships between positive psychological constructs and health outcomes in patients with cardiovascular disease: a systematic review. Int J Cardiol. 2015;195:265-80.

14. Schiavon CC, Marchetti E, Gurgel LG, Busnello FM, Reppold CT. Optimism and hope in chronic disease: a systematic review. Front Psychol. 2017;7:2022.

15. DuBois CM, Millstein RA, Celano CM, Wexler DJ, Huffman JC. Feasibility and acceptability of a positive psychological intervention for patients with type 2 diabetes. Prim Care Companion CNS Disord. 2016; doi:10.4088/PCC. $15 \mathrm{~m} 01902$. 
16. Baños RM, Espinoza M, García-Palacios A, Cervera JM, Esquerdo G, Barrajón E, et al. A positive psychological intervention using virtual reality for patients with advanced cancer in a hospital setting: a pilot study to assess feasibility. Support Care Cancer. 2013;21(1):263-70.

17. Rutter M. Psychosocial resilience and protective mechanisms. Am J Orthop. 1987:57(3):316-31

18. Strand EB, Zautra AJ, Thoresen M, Ødegård S, Uhlig T, Finset A. Positive affect as a factor of resilience in the pain-negative affect relationship in patients with rheumatoid arthritis. J Psychosom Res. 2006;60(5):477-84.

19. Li MY, Yang YL, Liu L, Wang L. Effects of social support, hope and resilience on quality of life among Chinese bladder cancer patients: a cross-sectional study. Health Qual Life Outcomes. 2016;14:73.

20. Nawaz A, Malik JA, Batool A. Relationship between resilience and quality of life in diabetics. J Coll Physicians Surg Pak. 2014;24(9):670-5.

21. Lee D, Cha B, Park CS, Kim BJ, Lee CS, Lee SJ, et al. Effects of resilience on quality of life in patients with bipolar disorder. J Affect Disord. 2016;207:434-41.

22. Rainone N, Chiodi A, Lanzillo R, Magri V, Napolitano A, Morra VB, et al. Affective disorders and health-related quality of life (HRQoL) in adolescents and young adults with multiple sclerosis (MS): the moderating role of resilience. Qual Life Res. 2017;26(3):727-36.

23. Min JA, Lee CU, Hwang SI, Shin Jl, Lee BS, Han SH, et al. The moderation of resilience on the negative effect of pain on depression and post-traumatic growth in individuals with spinal cord injury. Disabil Rehabil. 2014;36(14):1196-202.

24. Ye ZJ, Qiu HZ, Li PF, Liang MZ, Zhu YF, Zeng Z, et al. Predicting changes in quality of life and emotional distress in Chinese patients with lung, gastric, and colon-rectal cancer diagnoses: the role of psychological resilience. Psychooncology. 2017;26(6):829-35.

25. Wu WW, Tsai SY, Liang SY, Liu CY, Jou ST, Berry DL. The mediating role of resilience on quality of life and cancer symptom distress in adolescent patients with cancer. J Pediatr Oncol Nurs. 2015;32(5):304-13.

26. Chen HH, Chen DY, Chen YM, Lai KL. Health-related quality of life and utility: comparison of ankylosing spondylitis, rheumatoid arthritis, and systemic lupus erythematosus patients in Taiwan. Clin Rheumatol. 2017;36(1):133-42.

27. Prevoo ML, van 't Hof MA, Kuper HH, van Leeuwen MA, van de Putte LB, van Riel PL. Modified disease activity scores that include twenty-eight-joint counts. Development and validation in a prospective longitudinal study of patients with rheumatoid arthritis. Arthritis Rheum. 1995;38(1):44-8.

28. Block J, Kremen AM. IQ and ego-resiliency: conceptual and empirical connections and separateness. J Pers Soc Psychol. 1996;70(2):349-61.

29. Yang X, Zhao L, Wang L, Hao C, Gu Y, Song W, et al. Quality of life of transgender women from China and associated factors: a cross-sectional study. J Sex Med. 2016;13(6):977-87.

30. Wang Z, Yu H, Sun Y, Qin B. Correlation analysis of optimism, resilience and anxiety in lung cancer patients. J Chin Med Univ. 2017;46(3):238-40.

31. Hayes AF. An introduction to mediation, moderation, and conditional process analysis: a regression-based approach. New York: Guilford Press; 2013.

32. Zhu Y, Wang $Q$, Pang G, Lin L, Origasa $H$, Wang $Y$, et al. Association between body mass index and health-related quality of life: the "obesity paradox" in 21,218 adults of the Chinese general population. PLoS One. 2015;10(6):e0130613.

33. Uhlig T, Loge JH, Kristiansen IS, Kvien TK. Quantification of reduced healthrelated quality of life in patients with rheumatoid arthritis compared to the general population. J Rheumatol. 2007;34(6):1241-7.

34. Salaffi F, Sarzi-Puttini P, Girolimetti R, Atzeni F, Gasparini S, Grassi W. Healthrelated quality of life in fibromyalgia patients: a comparison with rheumatoid arthritis patients and the general population using the SF-36 health survey. Clin Exp Rheumatol. 2009;27(5 Suppl 56):67-74.

35. Ovayolu N, Ovayolu O, Karadag G. Health-related quality of life in ankylosing spondylitis, fibromyalgia syndrome, and rheumatoid arthritis: a comparison with a selected sample of healthy individuals. Clin Rheumatol. 2011;30(5):655-64.

36. Kwan YH, Koh ET, Leong KP, Wee HL, Tan Tock Seng Rheumatoid Arthritis Study Group. Association between helplessness, disability, and disease activity with health-related quality of life among rheumatoid arthritis patients in a multiethnic Asian population. Rheumatol Int. 2014;34(8):1085-93.

37. Karimi S, Yarmohammadian MH, Shokri A, Mottaghi P, Qolipour K, Kordi A, et al. Predictors and effective factors on quality of life among Iranian patients with rheumatoid arthritis. Mater Sociomed. 2013;25(3):158-62.

38. Ibn Yacoub Y, Amine B, Laatiris A, Hajjaj-Hassouni N. Health-related quality of life in Moroccan patients with rheumatoid arthritis. Clin Rheumatol. 2012; 31(10):1471-7.

39. Townes SV, Furst DE, Thenkondar A. The impact of tocilizumab on physical function and quality of life in patients with rheumatoid arthritis: a systematic literature review and interpretation. Open Access Rheumatol. 2012;4:87-92.

40. Zhao S, Chen Y, Chen $\mathrm{H}$. Sociodemographic factors associated with functional disability in outpatients with rheumatoid arthritis in Southwest China. Clin Rheumatol. 2015;34(5):845-51.

41. Salaffi F, Carotti M, Gasparini S, Intorcia M, Grassi W. The health-related quality of life in rheumatoid arthritis, ankylosing spondylitis, and psoriatic arthritis: a comparison with a selected sample of healthy people. Health Qual Life Outcomes. 2009;7:25.

42. Ding H, Han J, Zhang M, Wang K, Gong J, Yang S. Moderating and mediating effects of resilience between childhood trauma and depressive symptoms in Chinese children. J Affect Disord. 2017;211:130-5.

43. Wingo AP, Wrenn G, Pelletier T, Gutman AR, Bradley B, Ressler KJ. Moderating effects of resilience on depression in individuals with a history of childhood abuse or trauma exposure. J Affect Disord. 2010;126(3):411-4.

44. Ye Z, Chen L, Harrison SE, Guo H, Li X, Lin D. Peer victimization and depressive symptoms among rural-to-urban migrant children in China: the protective role of resilience. Front Psychol. 2016;7:1542.

45. Deshields TL, Heiland MF, Kracen AC, Dua P. Resilience in adults with cancer: development of a conceptual model. Psychooncology. 2016;25(1):11-8.

46. Johnston MC, Porteous T, Crilly MA, Burton CD, Elliott A, Iversen $L$, et al. Physical disease and resilient outcomes: a systematic review of resilience definitions and study methods. Psychosomatics. 2015;56(2):168-80.

47. Terrill AL, Molton IR, Ehde DM, Amtmann D, Bombardier CH, Smith AE, et al. Resilience, age, and perceived symptoms in persons with long-term physical disabilities. J Health Psychol. 2016;21(5):640-9.

48. Lu C, Yuan L, Lin W, Zhou Y, Pan S. Depression and resilience mediates the effect of family function on quality of life of the elderly. Arch Gerontol Geriatr. 2017;71:34-42

49. Graninger M. Behavioral training as additional therapy approach for rheumatoid arthritis. Z Rheumatol. 2015;74(7):579-83.

50. Sun J, Buys $N$, Jayasinghe R. Effects of community-based meditative tai chi programme on improving quality of life, physical and mental health in chronic heart-failure participants. Aging Ment Health. 2014;18(3):289-95.

\section{Submit your next manuscript to BioMed Central and we will help you at every step:}

- We accept pre-submission inquiries

- Our selector tool helps you to find the most relevant journal

- We provide round the clock customer support

- Convenient online submission

- Thorough peer review

- Inclusion in PubMed and all major indexing services

- Maximum visibility for your research

Submit your manuscript at www.biomedcentral.com/submit
) Biomed Central 